\title{
Design and Implementation of an Intelligent Mobile Game
}

\author{
Ekin Ekinci \\ Kocaeli University / Computer Engineering Department, Kocaeli, 41000, Turkey \\ Email: ekin.ekinci@kocaeli.edu.tr \\ Fidan Kaya Gülağız, Sevinç İlhan Omurca \\ Kocaeli University / Computer Engineering Department, Kocaeli, 41000, Turkey \\ Email: \{fidan.kaya, silhan\}@ kocaeli.edu.tr
}

\begin{abstract}
While the mobile game industry is growing with each passing day with the popularization of $3 \mathrm{G}$ smart devices, the creation of successful games, which may interest users, become quite important in terms of the survival of the designed game. Clustering, which has many application fields, is a successful method and its implementation in the field of mobile games is inevitable. In this study, classical ball blasting game was carried out based on clustering. In the game, clustering the color codes with K-means, Iterative K-means, Iterative Multi $\mathrm{K}$-means and K-medoids methods and blasting the balls of colors located in the same cluster by bringing them together were proposed. As a result of the experiments, the suitability of clustering methods for mobile based ball blasting game was shown. At the same time, the clustering methods were compared to produce the more successful clusters and because of obtaining more accurate results and stability, the use of $\mathrm{K}$-medoids method has been chosen for this game.
\end{abstract}

Index Terms-Mobile game, K-means, Iterative Kmeans, Iterative Multi K-means, K-medoids.

\section{INTRODUCTION}

Mobile games on mobile devices, which are provided to users via mobile communication network and described as electronic games, have significantly developed along with the indispensable spread of especially mobile devices with $3 \mathrm{G}$ support in our daily lives [1]. Mobile games have been able to sustain their development thanks to mobile features such as portability, ease of use and simplicity and user habits formed over time [2]. While they appealed to a certain number of users with the Snake game, Tetris game etc. at the end of 20th century, they have been one of the prominent platforms in game market on the world by accessing to 1.5 billion users around the world in 2014 according to the data of Global Mobile Game Industry [3].

It has been very important to appeal to users of thousands of games released by their developers every day, sustain their existence in the market and provide the qualifications to find a place in the market. Games are designed in accordance with certain rules. In order to design a successful game, these rules are based on entertaining the users with interesting scenarios and visual-audial features [4]. It is also possible to benefit from several disciplines for forming these rules. Games that can appeal to users' interests can be designed as mobile game by using clustering which is a data mining method and has several application fields.

Clustering, which is an unsupervised learning method because of the lack of identified clusters and accurate knowledge of the number of clusters [5], is described as categorizing objects in a data set into clusters according to their similarities based on a certain distance measure [6]. While the intra-cluster similarity is maximum in the formed clusters, the inter-cluster similarity is minimum. If these conditions are provided, clustering can be successful, otherwise, it is unsuccessful. Clustering, which is an important method in information inference with all these features, has several application fields such as pattern recognition, bio-information, text mining and machine learning $[7,8]$.

In this study, a ball blasting game consisting of three levels of difficulty was realized on a mobile cluster basis. 141 color codes were categorized into the primary colors red, green and blue and three parameters were obtained. Then, the decimal value was calculated for these parameters. K-means, Iterative K-means, Iterative Multi K-means and K-medoids clustering methods were realized on the calculated values. For each method, clusters were obtained with $\mathrm{k}=3, \mathrm{k}=4$ and $\mathrm{k}=5$. When three or more balls in the same cluster come together vertically or horizontally, they blast. Four evaluation measures were utilized for comparing the clustering methods. At the end of the experiments, it was aimed to choose the most appropriate clustering method for the mobile game by taking these evaluation measures into consideration. Since more successful clusters are obtained with K-medoids algorithm and this algorithm is more resolute, K-medoids algorithm was determined as the most appropriate algorithm for the game to be developed.

The remaining part of the study is organized as the following: In the $2^{\text {nd }}$ part, the clustering methods used are described. In the $3^{\text {rd }}$ part, evaluation measures for data sets and clustering algorithms are described. In the $4^{\text {th }}$ part, the experimental results are presented. In the $5^{\text {th }}$ 
part, the contributions of the study are given.

\section{METHOD}

Clustering is the unsupervised classification of patterns (observations, data items, or feature vectors) into groups (clusters) [9]. The only information clustering uses is similarity between data points .Many researchers [10] have been dealt with clustering problem in different disciplines. The clustering method can be divided into hierarchical and nonhierarchical [13]. Hierarchical clustering is a widely used data analysis technique. The idea is to build a binary tree of the data that successively merges similar groups of points. In this study, two different hierarchical clustering methods are used to cluster game data which are K-means and K-medoids. In this section the algorithms are explained in detailed.

\section{A. K-Means Algorithm}

$\mathrm{K}$-means algorithm is one of the widely used algorithms in data mining. It was developed by J.B. MacQueen [14] in 1967. Its aim is to separate the data set given to it as input into $k$ number of clusters. The $k$ parameter in the name of the algorithm stands for the cluster number to be obtained at the end of the clustering process. K-means algorithm works on the logic of forming clusters according to the distance to the point which is determined as center for each class. The distance of the objects of clusters to the central point also stands for the amount of error. The aim is to minimize the distance between objects of the cluster and maximize the distance between the clusters.

The algorithm consists of four main steps [15]: These are determining center of clusters, assigning the points out of the central point to clusters according to their distance to the center of clusters, calculation of the new center of clusters after each iteration and the iteration of processes till obtaining resolute center of clusters. The flowchart of K-means algorithm is presented in Fig. 1.

The biggest problem of K-means algorithm is determining the start point. If there is a bad choice at the beginning, changes will be too frequent in clustering process and in this situation, different clustering results will be obtained every time with the same number of iterations and different start points. Moreover, the algorithm may not provide good results if the dimensions and density of data groups are different and there are conflicts in the data. Besides, the complexity of K-means algorithm is less than the other clustering methods and realization of the algorithm is easier.

The time complexity of each iteration of $\mathrm{K}$-means algorithm is stated as $\mathrm{O}(n k d)$. Here the $n$ value stands for the number of records in the cluster, $k$ value stands for the number of clusters to be obtained and $d$ value stands for the number of features in each record. As it can be understood from the parameters, the dimension of data set, number of feature in the data and the dimensions of the iterations have an effect on process time, but even if when the method is applied to very big datasets, results can be obtained very quickly.
In this study, Iterative K-means and Iterative Multi Kmeans were also analyzed in addition to K-means method. Iterative $\mathrm{K}$-means algorithm works with minimum cluster number and maximum cluster number parameters to increase the accuracy of K-means algorithm. The method makes K-means algorithm work in minimum and maximum cluster number intervals and it calculates a score value for each cluster number. It gives the number of cluster which has the highest score value as a result. Iterative Multi K-means algorithm is a developed form of Iterative K-means algorithm in terms of iteration number. In a different way from Iterative K-means algorithm, it aims to obtain the best cluster number and the best iteration value for this cluster number by working with different numbers of iterations.

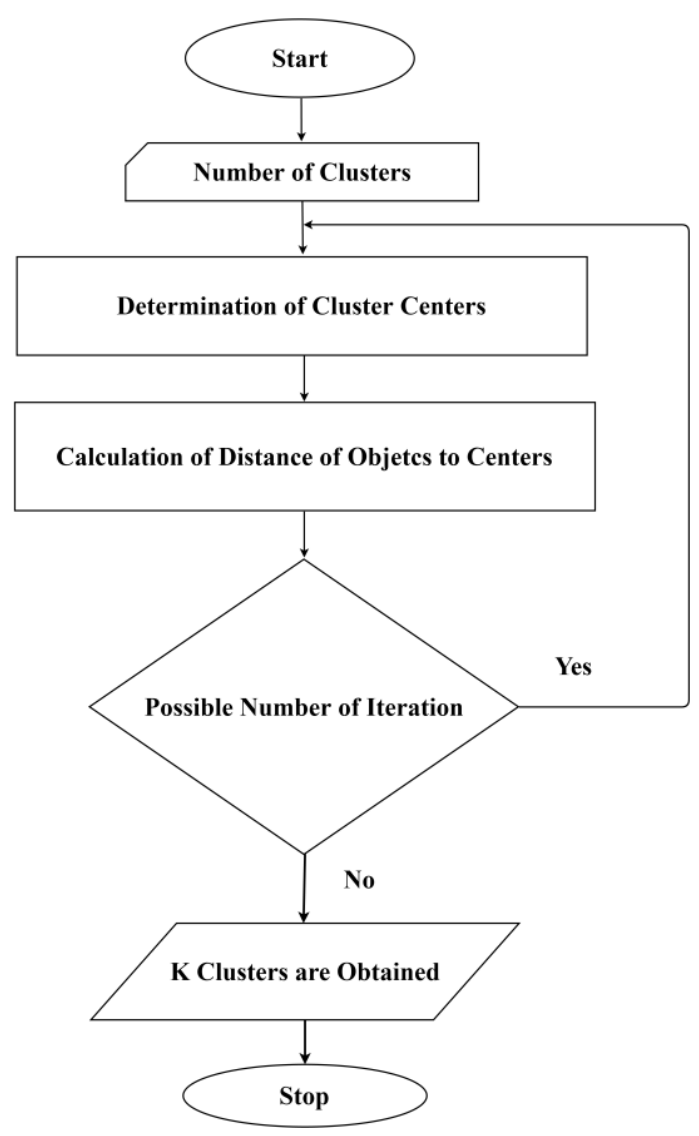

Fig.1. Flowchart of K-means algorithm.

\section{B. K-Medoids Algorithm}

K-medoids algorithm is based on the logic of finding $k$ number of representative objects which represent several structural features of data. For this reason, this method is called as K-medoids or PAM (Partitioning Around Medoids). The representative objects in the algorithm are called as medoids and these objects are in the closest point to the center of clusters. The most widely used Kmedoids algorithm was developed by Kaufman and Rousseeuw in 1987 [16]. The representative object is the most central object in the cluster and it makes the average distance to other objects minimum. Therefore, this division method is applied based on the logic of 
minimizing the total of dissimilarities between each object and its reference point.

The flowchart of K-medoids algorithm is presented in Fig. 2. After the $k$ number of representative objects is determined in this method, $k$ clusters are formed by assigning each object to the closest representative object [17]. In the next step, each representative object is replaced with a non-representative object and the replacement is carried on till the quality of the clustering is improved. In order to evaluate the quality of clustering, a function called as the cost function is used. This function calculates the similarity between an object and the cluster of this object. The decision process is conducted according to cost value of the function [18].

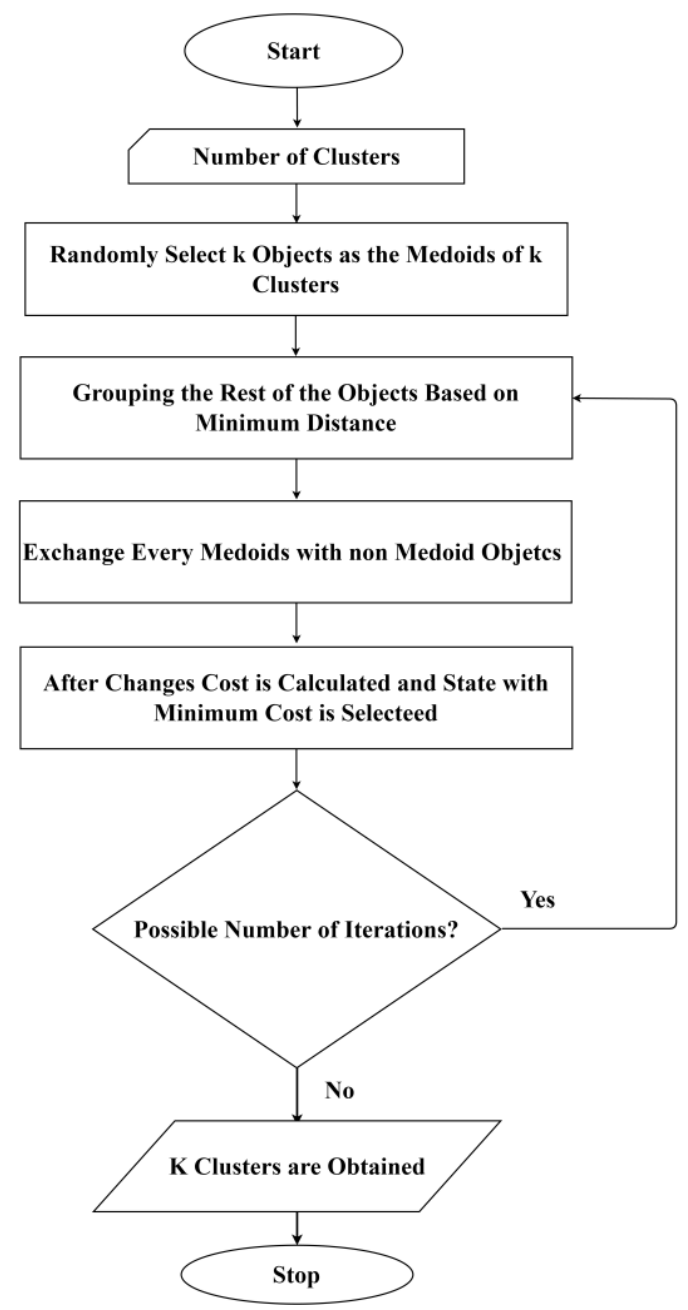

Fig.2. Flowchart of K-medoids algorithm.

The time complexity of each iteration of K-medoids algorithm is stated as $\mathrm{O}\left(k(n-k)^{2}\right)$. The $k$ parameter here stands for cluster number and $n$ parameter stands for the number of records in the data set. As it can be understood from the formula, K-medoids algorithm requires more place and time complexity than K-means algorithm. However, K-medoids algorithm provides more accurate results than $\mathrm{K}$-means and it is a resolute algorithm. It is different from K-means algorithm in that the first assignments to center of clusters have no effect on the clustering result in $\mathrm{K}$-medoids.

\section{Data SET AND Evaluation MeASures}

The data set used in this study was specially formed for the game to be developed. There are hexadecimal values of 141 color codes in the data set. These values were categorized into the primary colors red, green and blue before they were used for clustering and then, converted to decimal forms. The obtained color values with three parameters were saved in a different file. The clustering process was realized according to the distance values between these three parameters.

There are different methods developed for the evaluation of clustering algorithms. Some of these are listed as process time, sum of center of clusters similarities, (SOCS), sum of error squares (SSE), AIC (Akaike's Information Criterion) score and BIC (Bayesian Information Criterion) scores. Descriptions of these methods are given in Table 1.

Table 1. Methods Used for Evaluation of Clustering Algorithms

\begin{tabular}{|c|c|}
\hline Method & Description \\
\hline Process time & Refers to time it takes to cluster \\
\hline SOCS & Refers to sum of center of clusters similarities \\
\hline SSE & $\begin{array}{c}\text { Refers to sum of distance of points to center of } \\
\text { clusters in which they are found. }\end{array}$ \\
\hline AIC and BIC & $\begin{array}{c}\text { Refers to a measure of statistical model quality } \\
\text { over a given dataset }\end{array}$ \\
\hline
\end{tabular}

While applying process time and SOCS described in Table 1, any of the methods for distance calculation can be utilized. The formula of SSE calculation method is given in (1). The $k$ value in the formula stands for the cluster number, $c_{i}$ value $i$. stands for the center of clusters and $x$ value $i$. stands for the objects of cluster.

$$
S S E=\sum_{i=l}^{K} \sum x \in c_{i} \operatorname{distan} c e\left(x, c_{i}\right)^{2}
$$

The formula of AIC evaluation method is shown in (2) and the formula of BIC evaluation method is shown in (3). The $L$ value in (2) and (3) stands for the similarity calculation function used and the $p$ value stands for the number of features in the data set.

$$
A I C=-2 \log L+2 p
$$

The low AIC and BIC values show that the model is closer to reality and the use of these two parameters together is more efficient for the model or cluster number choices in evaluation.

$$
B I C=-2 \log L+\log (n) p
$$

The biggest advantage of AIC measure is that it is highly successful in both intra-cluster and inter-cluster evaluations. Both for the AIC and BIC equations, the second part of the equation calculate a penalty value for the elements that are included later. However, this value was determined as a low value for AIC while it is a 
higher value for BIC. With the high penalty value in the BIC equation, more accurate evaluation was aimed for big datasets.

In the context of the study, the comparison of clustering methods from different perspectives was conducted by the use of process time, SOCS and SSE evaluation methods. Java-ML (Java Machine Learning Library), which is the machine learning library of Java, was used for the realization of evaluation methods [19] and it was determined which method is more appropriate for the game to be developed.

\section{The Mobile Application DeVEloped}

The game developed is a simple ball blasting game. There are three levels of difficulty in the game. The difficulty levels are determined according to the dimension of the matrix appearing on the screen and the number of color clusters placed into the matrix. The difficulty levels of the game and the number of clusters corresponding to these difficulty levels are presented in Table 2.

The game realizes clustering process in the mobile environment according to the data set consisting of colors. When three or more balls in the same cluster come together vertically or horizontally, they blast. After the blast, the balls above replace the blasted balls and new balls in random colors are produced for the empty spaces at the top of the matrix. There is a time limit for each difficulty level in the game. Points are calculated considering the number of blasted balls and time taken to blast them. The screenshots of difficulty levels in the game are given in Fig. 3.

Table 2. Number of Clusters Corresponding to the Levels in the Game

\begin{tabular}{|c|c|c|c|}
\hline Difficulty Levels & Dimension of Matrix & Number of Clusters & Time \\
\hline Easy & $6 \times 6$ & 3 & 30 minutes \\
\hline Moderate & $9 \times 6$ & 4 & 25 minutes \\
\hline Difficult & $12 \times 6$ & 5 & 15 minutes \\
\hline
\end{tabular}
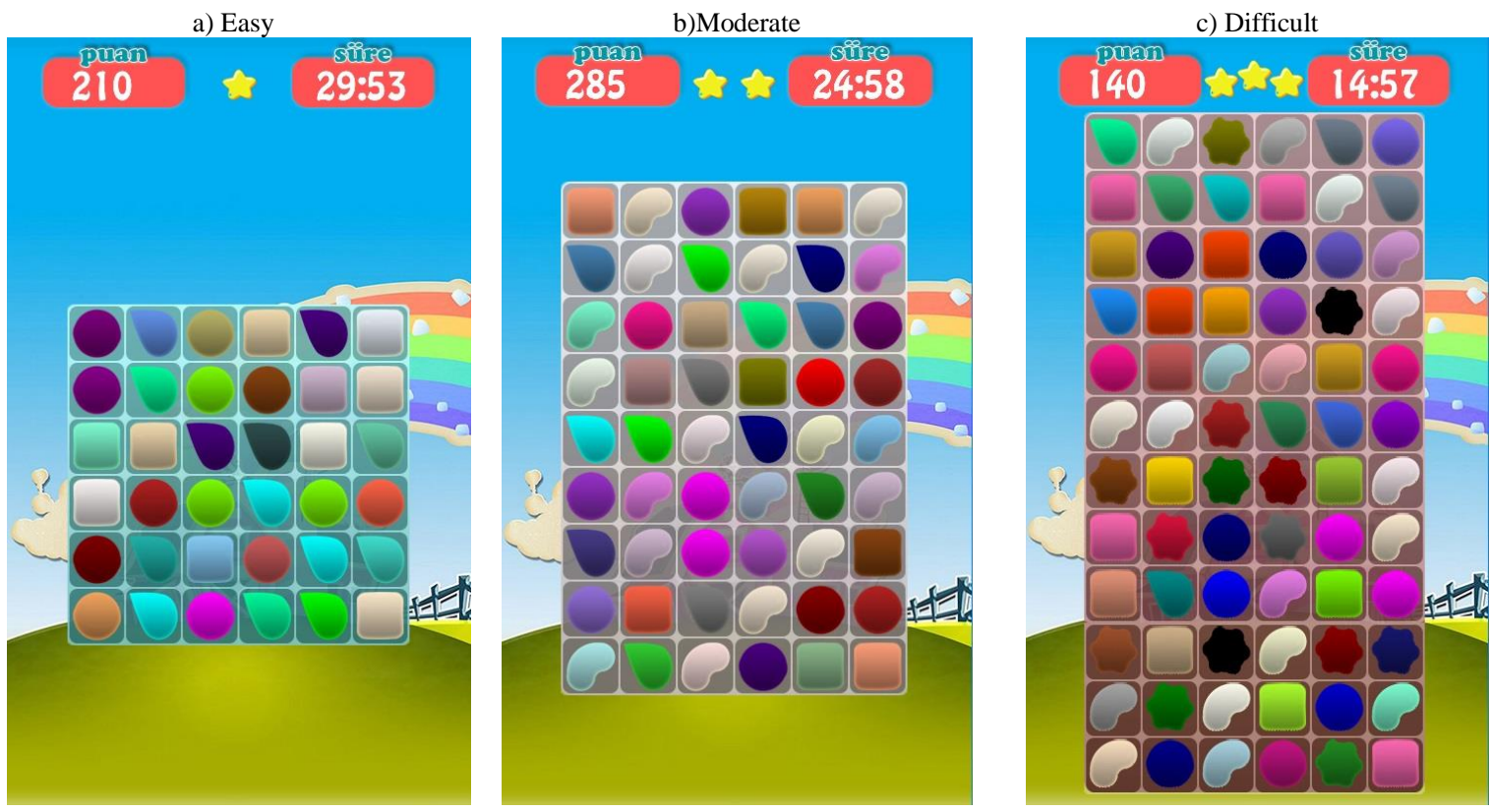

Fig.3. Screenshots of difficulty levels in the game

\section{EXPERIMENTAL RESULTS}

In order to understand whether clustering algorithms are sufficient for the game to be developed in terms of performance and accuracy, some tests were conducted. Experimental tests were realized on a computer on which Intel(R) Core(TM) i5-3317U CPU @ $1.70 \mathrm{GHz}, 8 \mathrm{~GB}$ RAM, 350 GB hard drive and Windows 7 Ultimate are uploaded.
It is necessary to choose the appropriate clustering method in order to obtain the cluster values mentioned in Table 2. For this reason, it was aimed to determine the most appropriate method by evaluating different clustering algorithms. The results obtained with the application of K-means algorithm on data set are presented in Table 3. There are process time and accuracy results of clusters obtained with different number of clusters in the table. When the process time column is examined, it is seen that the clustering process doesn't 
affect the performance of the game. The process times obtained are shorter than even one second and if we consider that the clustering process will be done only once at the beginning of the game, its effect on the performance is little if any.

Evaluation results of $\mathrm{K}$-means, Iterative $\mathrm{K}$-means and Iterative Multi K-means are given respectively in Table 3,
Table 4 and Table5. It was observed that the algorithms have similar performances in terms of process time, the center of clusters obtained and closeness to reality. Therefore, it is observed that the use of Iterative K-means or Iterative Multi K-means algorithms instead of $\mathrm{K}$ means algorithm will not cause a serious effect on the results.

Table 3. Evaluation Results of K-means Algorithm

\begin{tabular}{|c|c|c|c|c|c|}
\hline $\mathrm{k}$ & Process Time (sec.) & SOCS & SSE & AIC & BIC \\
\hline 2 & 0,000715 & 127.72 & 3913076.84 & 14430,869276 & 14431,018495 \\
\hline 3 & 0,000814 & 132.82 & 2780635.32 & 14286,456027 & 14286,605246 \\
\hline 4 & 0,000735 & 134.88 & 2299393.45 & 14172,267740 & 14172,267740 \\
\hline 5 & 0,000676 & 133.30 & 1908820.35 & 14083,520316 & 14083,669535 \\
\hline 10 & 0,000706 & 137.81 & 1036044.67 & 14013,227955 & 14013,377174 \\
\hline 15 & 0,001337 & 139.07 & 733983.47 & 14090,361464 & 14090,510683 \\
\hline
\end{tabular}

Table 4. Evaluation Results of Iterative K-means Algorithm

\begin{tabular}{|c|c|c|c|c|c|}
\hline $\mathrm{k}$ & Process Time (sec.) & SOCS & SSE & AIC & BIC \\
\hline 2 & 0,000721 & 127.32 & 3925900.84 & 14368,068617 & 14368,217837 \\
\hline 3 & 0,000738 & 132.82 & 2780635.32 & 14286,456027 & 14286,605246 \\
\hline 4 & 0,000705 & 133.14 & 2402667.46 & 14126,751763 & 14126,900982 \\
\hline 5 & 0,000816 & 135.52 & 1991643.28 & 14150,740182 & 14150,889401 \\
\hline 10 & 0,000691 & 138.03 & 1048043.83 & 14037,366551 & 14037,515770 \\
\hline 15 & 0,000709 & 137.61 & 828138.95 & 14024,403325 & 14024,552544 \\
\hline
\end{tabular}

Table 5. Evaluation Results of Iterative Multi K-means Algorithm

\begin{tabular}{|c|c|c|c|c|c|}
\hline $\mathrm{k}$ & Process Time (sec.) & SOCS & SSE & AIC & BIC \\
\hline 2 & 0,000814 & 127.73 & 3912928.79 & 14434,115946 & 14434,265165 \\
\hline 3 & 0,000737 & 132.82 & 2780635.32 & 14286,456027 & 14286,605246 \\
\hline 4 & 0,000695 & 135.46 & 2290333.21 & 14189,547951 & 14189,697170 \\
\hline 5 & 0,000783 & 133.52 & 1895319.41 & 14070,696099 & 14070,845318 \\
\hline 10 & 0,000726 & 138.29 & 1036810.57 & 14023,875312 & 14024,024532 \\
\hline 15 & 0,000762 & 138.93 & 734218.30 & 14082,862737 & 14083,011956 \\
\hline
\end{tabular}

Table 6. Evaluation Results of K-medoids Algorithm

\begin{tabular}{|c|c|c|c|c|c|}
\hline $\mathrm{k}$ & Process Time $(\mathrm{sec})$. & SOCS & SSE & AIC & BIC \\
\hline 2 & 0,001156 & 129.57 & 4171972.59 & 14683,482333 & 14683,631552 \\
\hline 3 & 0,001286 & 131.05 & 3777599.33 & 14665,799503 & 14665,948722 \\
\hline 4 & 0,000918 & 131.08 & 3628823.20 & 14662,039772 & 14662,188991 \\
\hline 5 & 0,001353 & 131.24 & 3611538.37 & 14710,335577 & 14710,484796 \\
\hline 10 & 0,001763 & 131.50 & 3422162.38 & 14782,823114 & 14782,972333 \\
\hline 15 & 0,001565 & 131.80 & 3391581.89 & 14911,419501 & 14911,568720 \\
\hline
\end{tabular}


When the results of K-medoids algorithm in Table 6 and other methods are compared, K-medoids algorithm requires a longer time for process time. At the same time, it is seen that the center of clusters calculated by $\mathrm{K}$ medoids algorithm, and therefore the results of clustering process is more accurate. When the results of the four different methods are evaluated, it is seen that all the methods are appropriate for mobile game programming; however, the methods have some minor advantages over one another. When we compared all algorithms, most accurate results were obtained by $\mathrm{K}$-medoids algorithm also K-medoids algorithm is a more stable than others. For these reasons K-medoids algorithm is used to develop proposed game.

\section{RESULTS}

In this study, which is developed to support mobile programming and the use of algorithms in mobile fields, it was revealed that the use of algorithms in mobile programming doesn't affect performance and more professional games can be developed. Moreover, it was shown that clustering algorithms carry out clustering process more quickly and accurately for the game developed.

This study also provided a simple comparison of clustering methods and showed which method is more appropriate to be used in which situations. As it can be understood from the experimental results, K-medoids algorithm is a more resolute algorithm than K-means algorithm; however, it is a more costly algorithm in terms of memory and process time. Since the data set used in this study is a small size data set, running the K-medoids algorithm didn't require a considerable time, but it will be more appropriate to choose faster clustering methods for big size data sets in mobile applications.

\section{REFERENCES}

[1] Yang, B., Zhang, Z.: Design and Implementation of High Performance Mobile Game on Embedded Device. Proceedings of the International Conference on Computer Application and System Modeling, Taiyuan, China, (2010).

[2] K. Klinger, Mobile Computing: Concepts, Methodologies, Tools, and Applications, $1^{\text {st }}$ ed. IGI Global, 2009.

[3] Newzoo Company. Link: https://newzoo.com/insights/tren d-reports/the-2015-gmgc-global-mobile-games-industrywh itebook/ (Last Access Date: 02.08.2016).

[4] Trisnadoli, A., Hendradjaya, B., Sunindyo, WD.: A Proposal of Quality Model for Mobile Game. Proceedings of the 5th International Conference on Electrical Engineering and Informatics, Bali, Indonesia, (2015).

[5] N. Doğru, A. Subaş1, "A Comparision of Clustering Techniques for Traffic Accident Detection," Turkish Journal of Electrical Engineering \& Computer Sciences, vol. 23, pp. 2124-2137, 2015.

[6] J. H. Chen, W. L. Hung, "An Automatic Clustering Algorithm for Probability Density Functions, ” Journal of Statistical Computation and Simulation, vol. 85, no. 15, pp. 304-3063, 2015.

[7] Bijuraj, L.V.: Clustering and Its Applications. Proceedings of the National Conference on New Horizons in IT, Mumbai, India, (2013).
[8] A.S. Shirkhorshidi, S. Aghabozorgi, T.Y. Wah, "A Comparison Study on Similarity and Dissimilarity Measures in Clustering Continuous Data," Plos One Journal, vol. 10, no. 12, 2015.

[9] A. K. Jain, M.N. Murty, P. J. Flynn, "Data Clustering: a Review," ACM Computing Surveys, vol. 31, no. 3, 1999.

[10] P.Jain, B. Buksh, "Accelerated K-means Clustering Algorithm," International Journal of Information Technology and Computer Science, vol. 8, no. 10, 2016.

[11] S. Chittineni, R. B. Bhogapathi, "Determining Contribution of Features in Clustering Multidimensional Data Using Neural Network," International Journal of Information Technology and Computer Science, vol. 4, no. 10, 2012.

[12] S. G. Rao, A. Govardhan, "Evaluation of H- and G-indices of Scientific Authors using Modified K-Means Clustering Algorithm," International Journal of Information Technology and Computer Science, vol. 8, no. 2, 2016.

[13] Y. Y. Shih, C. Y., Liu, "A method for customer lifetime value ranking - Combining the analytic hierarchy process and clustering analysis," Journal of Database Marketing \& Customer Strategy Management, vol. 11, no. 2, 2003.

[14] MacQueen, J.: Some Methods for Classification and Analysis of Multivariate Observation. Proceedings of the Fifth Berkeley Symposium on Mathematical Statistics and Probability, California, USA, (1967).

[15] Kaya, H., Köymen, K.: Veri Madenciliği Kavrami ve Uygulama Alanlari. Doğu Anadolu Bölgesi Araştırmaları, (2008).

[16] L. Kaufman, P.J. Rousseeuw, Statistical Data Analysis Based on the L1-Norm and Related Methods, Springer, 2002.

[17] L. Kaufman, P.J. Rousseeuw, Finding Groups in Data: An Introduction to Cluster Analysis, Wiley, 1990.

[18] M. Işık, A. Çanurcu, "K-ortalamalar, K-ortancalar ve Bulanik C-Means Algoritmalarının Uygulamalı Olarak Performanslarının Tespiti," İstanbul Ticaret Üniversitesi Fen Bilimleri Dergisi, vol. 6, no. 11, 2007.

[19] T. Abeel, Y.V. Peer, Y. Saeys, "Java-ML: A Machine Learning Library," Journal of Machine Learning Research, vol. 10, 2009.

\section{Authors' Profiles}

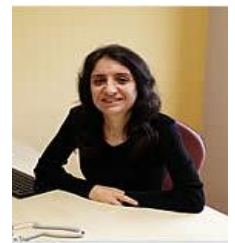

Ekin Ekinci has received her BEng. in Computer Engineering from Çanakkale Onsekiz Mart University in 2009 and ME. in Computer Engineering from Gebze Teknik University in 2012. She is currently working towards Ph.D. degree in Computer Engineering from Kocaeli University, Turkey. Also, she is currently a Research Assistant of Computer Engineering Department at Kocaeli University in Turkey. Her main research interests include text mining, sentiment analysis, natural language processing and machine learning.

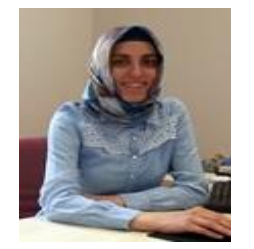

Fidan Kaya Gülağız has received her BEng. in Computer Engineering from Kocaeli University in 2010 and ME. in Computer Engineering from Kocaeli University in 2012. She is currently working towards Ph.D. degree in Computer Engineering from Kocaeli University, Turkey. Also, she is currently a Research Assistant of Computer Engineering 
Department at Kocaeli University in Turkey. Her main research interests include data synchronization, distributed file systems and data filtering methods.

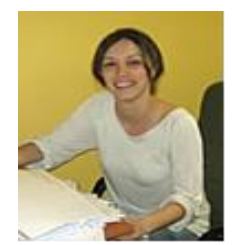

Sevinç İlhan Omurca was born in Burdur in Turkey at 23th December 1979. She is an associate professor at the Kocaeli University Computer Engineering Department in Turkey. She has a Ph.D. at the Kocaeli University Electronics and Communications Engineering. Her main research interests include text mining, sentiment analysis, natural language processing, machine learning and data mining.

How to cite this paper: Ekin Ekinci, Fidan Kaya Gülağız, Sevinç İlhan Omurca,"Design and Implementation of an Intelligent Mobile Game", International Journal of Modern Education and Computer Science(IJMECS), Vol.9, No.3, pp.10-16, 2017.DOI: 10.5815/ijmecs.2017.03.02 